\title{
ON THE SUMMABILITY OF FOURIER SERIES
}

\author{
BY W. C. RANDELS
}

1. Introduction. It is well known that the Abel method of summability is stronger than the Cesàro methods of any order. An example has been given* to show that there are series which are Abel summable but not Cesàro summable for any order. This series is one for which $a_{n} \neq o\left(n^{\alpha}\right)$ for any $\alpha$, and hence which cannot be $(C, \alpha)$ summable for any $\alpha$. This series cannot be a Fourier series since for all Fourier series $a_{n}=o(1)$. We propose to give an example of the existence of a Fourier series which is Abel summable but not Cesàro summable.

We shall make use of some results of Paley $\dagger$ which show that, if the Fourier series of $f(x)$,

$$
\frac{a_{0}}{2}+\sum_{n=1}^{\infty}\left(a_{n} \cos n x+b_{n} \sin n x\right),
$$

is $(C, \alpha)$ summable at the point $x$, then, for $\beta>\alpha$,

$$
\begin{aligned}
R_{\beta}(f, t) & =\beta \int_{0}^{t}\{f(x+\tau)+f(x-\tau)-2 f(x)\}(t-\tau)^{\beta-1} d \tau \\
& =o\left(t^{\beta}\right), \quad \text { as } \quad t \rightarrow 0,
\end{aligned}
$$

and conversely, if $R_{\alpha}(f, t)=o\left(t^{\alpha}\right)$, as $t \rightarrow 0$, then the series (1) is $(C, \beta)$ summable for every $\beta>\alpha+1$. We shall first show that for every $n>1$ there is a function $f_{n}(x)$ such that at $x=0$

$$
\varlimsup_{t \rightarrow 0}\left|\frac{1}{t^{j}} R_{j}\left(f_{n}, t\right)\right|=\infty, \quad(j \leqq n-1),
$$

but

$$
R_{n}\left(f_{n}, t\right)=o\left(t^{n}\right), \quad \text { as } \quad t \rightarrow 0 .
$$

This implies that the Fourier series of $f_{n}(x)$ is $(C, n+2)$ summable at $x=0$ and therefore Abel summable. The function

* See Landau, Darstellung und Begründung einiger neuer Ergebnisse der Funktionentheorie, 1929, p. 51.

$\dagger$ R. E. A. C. Paley, On the Cesàro summability of Fourier series and allied series, Proceedings of the Cambridge Philosophical Society, vol. 26 (1929), pp. 173-203. 


$$
f(x)=\sum_{n=2}^{\infty} d_{n} f_{n}(x)
$$

is then defined with the $d_{n}$ 's so chosen that the Fourier series of $f(x)$ is Abel summable, but for every $n$

$$
R_{n}(f, t) \neq o\left(t^{n}\right), \quad \text { as } \quad t \rightarrow 0 .
$$

This implies, by the theorem of Paley, that the Fourier series of $f(x)$ cannot be $(C, \alpha)$ summable for any $\alpha$.

2. Properties of $f_{n}(x)$. We suppose for the moment that $n$ is fixed and we let $c=(1+1 /(n-1 / 2))$. We define $a_{\nu}=2^{-c \nu}$, $b_{\nu}=2^{-\nu}-a_{\nu}$; then, if $\nu \geqq n, b_{\nu}>2^{-(\nu+1)}$, so that the intervals $\left(b_{\nu}, 2^{-\nu}\right)$ are non-overlapping for $\nu \geqq n$. We define

$$
f_{n}(x)=\left\{\begin{array}{l}
2^{\nu}, b_{\nu} \leqq|x| \leqq b_{\nu}+\frac{a_{\nu}}{2^{n}}, \quad(\nu=n, n+1, \cdots), \\
-f_{n}\left(x-2^{j} \frac{a_{\nu}}{2^{n}}\right), \quad b_{\nu}+2^{j} \frac{a_{\nu}}{2^{n}}<|x| \leqq b_{\nu}+2^{j+1} \frac{a_{\nu}}{2^{n}} \\
\quad(j=0, \cdots, n-1 ; \nu=n, \cdots), \\
0, \text { elsewhere on }(-\pi, \pi) .
\end{array}\right.
$$

Then $f_{n}(x) \subset L$ on $(-\pi, \pi)$, for

$$
\int_{-\pi}^{\pi}\left|f_{n}(x)\right| d x=2 \sum_{\nu=n}^{\infty} 2^{\nu} a_{\nu}=2 \sum_{\nu=n}^{\infty} 2^{-\nu /(n-1 / 2)}<\infty .
$$

At $x=0, f_{n}(x+t)+f_{n}(x-t)-2 f_{n}(x)=2 f_{n}(t)$. We have

$$
\int_{b_{\nu}}^{b_{\nu}+2\left(a_{\nu} / 2^{n}\right)} f_{n}(t) d t=\int_{b_{\nu}}^{b_{\nu}+a_{\nu} / 2^{n}} f_{n}(t) d t-\int_{b_{\nu}}^{b_{\nu}+a_{\nu} / 2^{n}} f_{n}(t) d t=0 .
$$

By the definition of $f_{n}(x)$,

$$
f_{n}(t)=-f\left(t-2^{j} \cdot \frac{a_{\nu}}{2^{n}}\right), \quad b_{\nu}+2^{j} \frac{a_{\nu}}{2^{n}}<t \leqq b_{\nu}+2^{j} \frac{a_{\nu}}{2^{n}},
$$

so that by induction

$$
\int_{b_{\nu}}^{b_{\nu}+2^{j}\left(a_{\nu} / 2^{n}\right)} f_{n}(t) d t=0, \quad(1 \leqq j \leqq n) ;
$$


and therefore, if $b_{\nu}+2^{j}\left(a_{\nu} / 2^{n}\right)<t,(1 \leqq j \leqq n-1)$,

$$
R_{1}\left(f_{n}, t\right)=2 \int_{b_{\nu}+2^{j}\left(a_{\nu} / 2^{n}\right)}^{t} f_{n}(\tau) d \tau .
$$

Hence, if $b_{\nu}+2^{j} 2\left(a_{\nu} / 2^{n}\right)<t<b_{\nu}+2^{j} 2\left(a_{\nu} / 2^{n}\right),(0 \leqq j \leqq n-2)$,

$$
R_{1}\left(f_{n}, t\right)=-R_{1}\left(f_{n}, t-2^{j} \cdot 2 \cdot \frac{a_{\nu}}{2^{n}}\right)
$$

Since

$$
R_{k+1}\left(f_{n}, t\right)=(k+1) \int_{0}^{t} R_{k}\left(f_{n}, \tau\right) d \tau,
$$

we see that in the same way, if $t>b_{\nu}$,

$$
\frac{1}{k+1} R_{k+1}\left(f_{n}, t\right)=\int_{b_{\nu}}^{t} R_{k}\left(f_{n}, \tau\right) d \tau,
$$

and, for

$$
\begin{gathered}
b_{\nu}+2^{j} \cdot 2^{k+1}\left(a_{\nu} / 2^{n}\right)<t<b_{\nu}+2^{j+1} \cdot 2^{k+1}\left(a_{\nu} / 2^{n}\right),(j+k \leqq n-2), \\
R_{k+1}\left(f_{n}, t\right)=-R_{k+1}\left(f_{n}, t-2^{i} \cdot 2^{k+1} \cdot \frac{a_{\nu}}{2^{n}}\right) .
\end{gathered}
$$

Therefore, for $k \leqq n-1$,

$$
\begin{aligned}
R_{k}\left(f_{n}, b_{\nu}+\frac{a_{\nu}}{2^{n}}\right) & =2 k 2^{\nu} \int_{0}^{a_{\nu} / 2^{n}}\left(\frac{a_{\nu}}{2^{n}}-t\right)^{k-1} d t \\
& =2^{\nu+1}\left(\frac{a_{\nu}}{2^{n}}\right)^{k} \neq o\left(2^{\nu k}\right) \text { as } \quad \nu \rightarrow \infty
\end{aligned}
$$

Finally, if $b_{\nu} \leqq t<2^{-\nu}$,

$$
\begin{aligned}
R_{n}\left(f_{n}, t\right) & =2 n \int_{0}^{t} f_{n}(\tau)(t-\tau)^{n-1} d \tau=O\left(2^{\nu} \int_{b_{\nu}}^{t}(t-\tau)^{n-1} d \tau\right) \\
& =O\left(2^{\nu} a_{\nu}{ }^{n}\right)=O\left(2^{\nu} 2^{-n \nu /(n-1 / 2)} 2^{-n \nu}\right)=o\left(2^{-n \nu}\right)=o(t) \text { as } t \rightarrow 0 .
\end{aligned}
$$

Therefore the function $f_{n}(x)$ has the properties (2) and (3).

3. A Function whose Fourier Series is not Summable $(C, \alpha)$. As we have already mentioned, the Fourier series of $f_{n}(x)$ will be Abel summable at $x=0$. Therefore, 


$$
\begin{aligned}
& A_{n}=\underset{0 \leqq r<1}{1 . \mathrm{u} . \mathrm{b} .} A\left(f_{n}, r\right) \\
& =\underset{0 \leqq r<1}{\operatorname{lou} . \mathrm{b}} \frac{1}{2 \pi} \int_{0}^{\pi}\left\{f_{n}(x+t)+f_{n}(x-t)-2 f_{n}(x)\right\} \frac{1-r^{2}}{1-2 \cos t+r^{2}} d t
\end{aligned}
$$

will exist. We may define two sequences $\left\{d_{n}\right\}$ and $\left\{t_{n}\right\}$ simultaneously by induction so that

$$
d_{n} \leqq \min \left(\frac{1}{2^{n} A_{n}}, \frac{1}{2^{n}}, \frac{1}{2^{n} \int_{-\pi}^{\pi}\left|f_{n}(t)\right| d t}\right)
$$

$$
\begin{array}{ll}
\text { (5) } d_{n} \leqq \frac{1}{2^{n}} \min _{\nu \leqq n-2}\left(\frac{1}{t_{\nu+1}^{-\nu} R_{\nu}\left(f_{n}, t_{\nu+1}\right)}\right), \\
\text { (6) }\left|t_{n}^{-(n-1)} R_{n-1}\left(f_{n}, t_{n}\right)\right|>\frac{n}{d_{n}}, \\
\text { (7) }\left|t_{n}^{-(n-1)} R_{n-1}\left(f_{n}, t_{n}\right)\right|<\frac{1}{n}, \quad(\nu \leqq n-1) .
\end{array}
$$

It is clear that $d_{n}$ can be chosen so as to satisfy (4) and (5). It is possible to choose $t_{n}$ satisfying (6) and (7), since

and

$$
\varlimsup_{t \rightarrow 0}\left|t^{-(n-1)} R_{n-1}\left(f_{n}, t\right)\right|=\infty,
$$

$$
t^{-\mu} R_{\mu}\left(f_{n}, t\right)=o(1) \quad \text { as } \quad t \rightarrow 0, \quad \text { for } \quad \mu \geqq n .
$$

The function

$$
f(x)=\sum_{n=2}^{\infty} d_{n} f_{n}(x)
$$

is integrable, for

$$
\int_{-\pi}^{\pi}|f(x)| d x \leqq \sum_{n=2}^{\infty} d_{n} \int_{-\pi}^{\pi}\left|f_{n}(x)\right| d x \leqq \sum_{n=2}^{\infty} 2^{-n} .
$$

The Fourier series of $f(x)$ is Abel summable, since

$$
A(f, r)=\sum_{n=2}^{\infty} d_{n} A\left(f_{n}, r\right)
$$

and $d_{n} A\left(f_{n}, r\right) \leqq 1 / 2^{n}$, and $A\left(f_{n}, r\right) \rightarrow 0$ as $r \rightarrow 1$, which implies that $A(f, r) \rightarrow 0$ as $r \rightarrow 1$. 
We shall show that, for every $n, R_{n}(f, t) \neq o\left(t^{n}\right)$, as $t \rightarrow 0$. Let us suppose that, for some $n, R_{n}(f, t)=o\left(t^{n}\right)$, as $t \rightarrow 0$; then, since

$$
R_{n+1}(f, t)=(n+1) \int_{0}^{t} R_{n}(f, \tau) d \tau,
$$

there would be a constant $K$ such that for all $t$ and $m \geqq n$ we would have

$$
\left|R_{m}(f, t)\right| \leqq K t^{m}
$$

We shall show that for every $n$

$$
\left|t_{n}^{-(n-1)} R_{n-1}\left(f, t_{n}\right)\right|>n+o(1), \quad \text { as } \quad n \rightarrow \infty,
$$

which contradicts $(8)$. We have

$$
\begin{aligned}
t_{n}^{-(n-1)} R_{n-1}\left(f, t_{n}\right)= & \sum_{\nu=2}^{\infty} d_{\nu} t_{n}^{-(n-1)} R_{n-1}\left(f_{\nu}, t_{n}\right) \\
= & \sum_{\nu=2}^{n-1} d_{\nu} t_{n}^{-(n-1)} R_{n-1}\left(f_{\nu}, t_{n}\right)+d_{n} t_{n}^{-(n-1)} R_{n-1}\left(f_{n}, t_{n}\right) \\
& +\sum_{\nu=n+1}^{\infty} d_{\nu} t_{n}^{-(n-1)} R_{n-1}\left(f_{\nu}, t_{n}\right) .
\end{aligned}
$$

By (7),

$$
\left|\sum_{\nu=2}^{n-1} d_{\nu} t_{n}^{-(n-1)} R_{n-1}\left(f_{\nu}, t_{n}\right)\right|<\frac{1}{n} \sum_{\nu=2}^{n-1}\left|d_{\nu}\right|=o(1), \quad \text { as } \quad n \rightarrow \infty,
$$

and, by (5),

$$
\left|\sum_{\nu=n+1}^{\infty} d_{\nu} t_{n}^{-(n-1)} R_{n-1}\left(f_{\nu}, t_{n}\right)\right| \leqq \sum_{\nu=n+1}^{\infty} 2^{-n}=o(1), \quad \text { as } \quad n \rightarrow \infty,
$$

so that, by $(6)$,

$$
\begin{aligned}
\left|t_{n}^{-(n-1)} R_{n-1}\left(f, t_{n}\right)\right| & =\left|d_{n} t_{n}^{-(n-1)} R_{n-1}\left(f_{n}, t_{n}\right)\right|+o(1) \\
& >n+o(1), \quad \text { as } n \rightarrow \infty .
\end{aligned}
$$

Therefore by the theorem of Paley the Fourier series of $f(x)$ cannot be $(C, \alpha)$ summable for any $\alpha$.

NORTHWESTERN UNIVERSITY 